\title{
Delayed detonations in full-star models of type la supernova explosions
}

\author{
F. K. Röpke ${ }^{1,2}$ and J. C. Niemeyer ${ }^{3}$ \\ 1 Max-Planck-Institut für Astrophysik, Karl-Schwarzschild-Str. 1, 85741 Garching, Germany \\ 2 Department of Astronomy and Astrophysics, University of California, Santa Cruz, CA 95064, USA \\ ${ }^{3}$ Lehrstuhl für Astronomie, Universität Würzburg, Am Hubland, 97974 Würzburg, Germany
}

Received 18 October 2006 / Accepted 12 December 2006

\section{ABSTRACT}

\begin{abstract}
Aims. We present the first full-star three-dimensional explosion simulations of thermonuclear supernovae including parameterized deflagration-to-detonation transitions that occur once the flame enters the distributed burning regime.

Methods. Treating the propagation of both the deflagration and the detonation waves in a common front-tracking approach, the detonation is prevented from crossing ash regions.

Results. Our criterion triggers the detonation wave at the outer edge of the deflagration flame and consequently it has to sweep around the complex structure and to compete with expansion. Despite the impeded detonation propagation, the obtained explosions show reasonable agreement with global quantities of observed type Ia supernovae. By igniting the flame in different numbers of kernels around the center of the exploding white dwarf, we set up three different models shifting the emphasis from the deflagration phase to the detonation phase. The resulting explosion energies and iron group element productions cover a large part of the diversity of type Ia supernovae.
\end{abstract}

Conclusions. Flame-driven deflagration-to-detonation transitions, if hypothetical, remain a possibility deserving further investigation.

Key words. stars: supernovae: general - hydrodynamics - instabilities - turbulence - methods: numerical

\section{Introduction}

The observables of type Ia supernovae ( $\mathrm{SNe}$ Ia) are determined by the composition of the ejecta and the energy release in the explosion. Both depend sensitively on the density at which the carbon-oxygen white dwarf (WD) material is burned by the thermonuclear flame. This, in turn, is a consequence of the competition between the flame propagation and the expansion of the WD due to nuclear energy release. Therefore, reproducing the characteristics of SNe Ia in a thermonuclear white dwarf explosion model requires a specific sequence of flame velocities.

After the thermonuclear flame has ignited near the center of the WD, two distinct modes of outward propagation are hydrodynamically admissible. In a subsonic deflagration the flame is mediated by the thermal conduction of the degenerate electrons, while a supersonic detonation is driven by shock waves. Observational constraints rule out a prompt detonation (Arnett 1969) and thus the flame has to start out as a deflagration (Nomoto et al. 1976). Such a scenario leads to a stratification of dense fuel atop light ashes. Due to Rayleigh-Taylor and KelvinHelmholtz instabilities, turbulence is generated. This results in a turbulent cascade with eddies decaying into ever smaller ones until they are dissipated at the Kolmogorov scale ( $\$ 1 \mathrm{~mm})$. In the cascade, the turbulent velocity decreases with length scale. Thus, turbulent motions interact with the flame down to the so-called Gibson length $l_{\mathrm{G}}$, where the turbulent velocity fluctuations $v^{\prime}$ become comparable to the laminar flame speed $s_{1}$,

$v^{\prime}\left(l_{\mathrm{G}}\right) \approx s_{1}$.

For most parts of the explosion, $l_{\mathrm{G}}$ is much larger than the flame width $l_{\mathrm{f}}$, indicating that the internal flame structure is unaffected by turbulence. Hence the interaction in this flamelet regime of turbulent combustion is purely kinematic, corrugating the flame and accelerating it by increasing its surface. However, as the WD star expands, the fuel density drops, $s_{1}$ decreases, and $l_{\mathrm{f}}$ increases (Timmes \& Woosley 1992). As soon as $l_{\mathrm{G}} \lesssim l_{\mathrm{f}}$, turbulent eddies distort the internal flame structure. This distributed burning regime commences when the fuel density has dropped close to values at which deflagration burning ceases (Niemeyer \& Woosley 1997; Niemeyer \& Kerstein 1997; Röpke \& Hillebrandt 2005b).

In order to burn significant fractions of the WD, a high flame propagation speed is necessary. Even a turbulence-boosted flame is relatively slow so that it may not lead to a sufficient fuel consumption before fuel densities drop below the burning threshold $\left(\$ 10^{7} \mathrm{~g} \mathrm{~cm}^{-3}\right)$. Although turbulent deflagration models (Reinecke et al. 2002b; Gamezo et al. 2003; Röpke \& Hillebrandt 2005a; Röpke et al. 2006; Schmidt \& Niemeyer 2006) reproduce the observational features of weak SN Ia explosions (Blinnikov et al. 2006), there exist open questions (Kozma et al. 2005) and presently it seems that they cannot account for the more energetic events. This issue certainly needs more exploration (in particular the buoyancy induced turbulence effects at the high Reynolds numbers of $\sim 10^{14}$ expected in SNe Ia, see Cabot \& Cook 2006) but it may also be interpreted as an incompleteness of such models. If more burning is necessary than the deflagration model allows for, a deflagration-to-detonation transition (DDT) would provide the ultimate flame acceleration. The problem of such delayed detonation models (Khokhlov 1991; Woosley \& Weaver 1994) is the absence of a robust physical mechanism that allows predicting a DDT in thermonuclear supernova explosions (Niemeyer 1999; Röpke et al. 2004a,b). 
Therefore invoking a DDT makes it necessary to introduce a free parameter into the model.

Examples of such parametrized models were recently given. Gamezo et al. (2005) assume the DDT to take place at an arbitrarily chosen instant and location, while Golombek \& Niemeyer (2005) impose a DDT once the flame enters the distributed burning regime.

Here we present the first three dimensional simulations of delayed detonations based on the numerical methods proposed by Golombek \& Niemeyer (2005) and adopting their DDT parametrization. Since the only instant where the flame properties change drastically is the beginning of the distributed burning regime, it seems natural to assume a DDT here (Niemeyer \& Woosley 1997). Although presently there is no indication of a DDT occurring in the distributed burning regime (Lisewski et al. 2000; Zingale et al. 2005), more exploration is necessary to settle this question. Our DDT parametrization does not a priori fix the instant of its occurrence nor its location on the flame and thus a failure triggering a viable detonation is possible. If successful, however, the detonation propagation is likely to proceed asymmetrically. In order to avoid artificial symmetries in the setup of the simulations, we pursue a full-star implementation of the model. Our aim is to determine whether such a model is consistent with global properties of observed $\mathrm{SNe} \mathrm{Ia}$, which is non-trivial since here the DDT is not used to tune the model but results from a physically motivated hypothesis.

\section{Modeling approach}

Aiming at parameterizing the location and time of the DDT according to properties of the deflagration flame, a self-consistent treatment of the interaction of the flame with turbulence is essential. This is achieved by a large-eddy simulation approach on the basis of methods presented by Niemeyer \& Hillebrandt (1995), Reinecke et al. (1999), Reinecke et al. (2002a), Röpke (2005), and Schmidt et al. (2006). In particular, the deflagration flame is represented as a sharp interface between fuel and ashes applying the level set method and unresolved turbulence is described via a subgrid-scale model from which the turbulent flame propagation speed is determined.

Maier \& Niemeyer (2006) found that even tiny regions of ash stall the detonation wave. Consequently, the detonation has to sweep around the complex deflagration structure. This requires a versatile implementation of the detonation. Following the suggestion of Golombek \& Niemeyer (2005) we model the detonation wave in the same approach as the deflagration flame applying a second level set treatment (see this reference for thorough tests of the implementation).

Lacking a sound theoretical understanding of the mechanism of the DDT, we restrict our model to a single DDT which is achieved by initiating the detonation level set the first patch of the deflagration flame where $l_{\mathrm{f}} \geq 0.98 l_{\mathrm{G}}$. Although this approach may seem rather artificial, it was chosen to simplify matters and to obtain a clear picture of the detonation propagation through the WD. Physically, it is motivated by the assumption that a DDT is a rare event.

In fuel regions the propagation speed of the detonation front is prescribed according to Sharpe (1999), while propagation into ash is suppressed. One question to be addressed in the present study is to which degree such a handicapped detonation is capable of burning major parts of the WD matter - an issue calling for three-dimensional simulations.

Our description of the nuclear reactions is based on Reinecke et al. (2002a) with the exception that the lower thresholds are set differently for burning $\mathrm{C}$ and $\mathrm{O}$, following Khokhlov (1991). While we allow C consumption down to fuel densities of $10^{6} \mathrm{~g} \mathrm{~cm}^{-3}, \mathrm{O}$ consumption ceases already at $10^{7} \mathrm{~g} \mathrm{~cm}^{-3}$. The WD is constructed using a suitable equation of state assuming isothermality at $T=5 \times 10^{5} \mathrm{~K}$, a central density of $2.9 \times 10^{9} \mathrm{~g} \mathrm{~cm}^{-3}$, and a composition of equal parts by mass of $\mathrm{C}$ and $\mathrm{O}$. It is set up on a Cartesian computational grid of $256^{3}$ cells with outflow boundary conditions on all sides. As described by Röpke et al. (2006), we follow the propagation of the deflagration flame and the WD expansion with two nested moving grid patches optimizing the flame resolution.

\section{Simulations}

With the DDT parametrization fixed as above, the only major undetermined parameter that impacts the evolution of the model is the configuration of the deflagration flame ignition. Although not attempting a thorough exploration of the parameter space here, we set up three different models in the multispot scenario similar to the simulations presented by Röpke et al. (2006). This allows us to test the robustness of the imposed DDT criterion. By placing 5 (Model DD_005), 20 (Model DD_020), and 800 (Model DD_800) flame ignition kernels inside a radius of $180 \mathrm{~km}$ around the WD's center, the efficiency of burning in the deflagration phase is significantly altered. While Model DD_005 leads to relatively little burning, igniting 800 flames results in a strong deflagration phase. We focus our description on the intermediate case of Model DD_020.

After ignition, the deflagration phase proceeds in a similar way as described by Röpke et al. (2006). The burning bubbles grow by flame propagation and rise buoyantly towards the surface of the WD. Due to instabilities and partial merger of the bubbles, a complex connected structure develops. The parametrized DDT criterion is met first $0.724 \mathrm{~s}$ after ignition at the outer edge of the flame front. Here, the detonation is triggered by initiating the corresponding level set, as shown in the upper panel of Fig. 1. The fact that the outer edge of the deflagration flame is chosen by our DDT criterion is not surprising. Turbulence is generated preferentially at large buoyant bubbles and at the same time the density is lowest at the outermost parts of the flame making the flame broadest here. This favors the transition to the distributed burning regime and thus the parametrized initiation of the detonation wave.

The center and lower panels of Fig. 1 show the propagation of the detonation wave. As implemented in our model, it cannot cross ash regions and, burning towards the star's center, it therefore wraps around the corrugated deflagration structure. In this way, it takes about $0.2 \mathrm{~s}$ before it arrives at the center of the WD. Meanwhile, the star keeps expanding and the deflagration continues in regions not yet reached by the detonation wave. Consequently, the density of the fuel ahead of the detonation drops quickly after passing the WD's center. In Model DD_020 burning stalls shortly before the detonation reaches the far side of the deflagration structure. This still implies burning of most of the WD, since these deflagration features have already reached the low density edge of the star.

The DDT parameters and the results of all three simulations simulations are summarized in Table 1 . The more kernels the deflagration is ignited in, the less energetic becomes the explosion and the less NSE elements are synthesized. The latter effect is partially compensated by more IME produced in weaker explosions giving rise to comparable masses of total burned material. In terms of the masses of the explosion products, the entire fuel remaining from the deflagration is affected by the detonation 

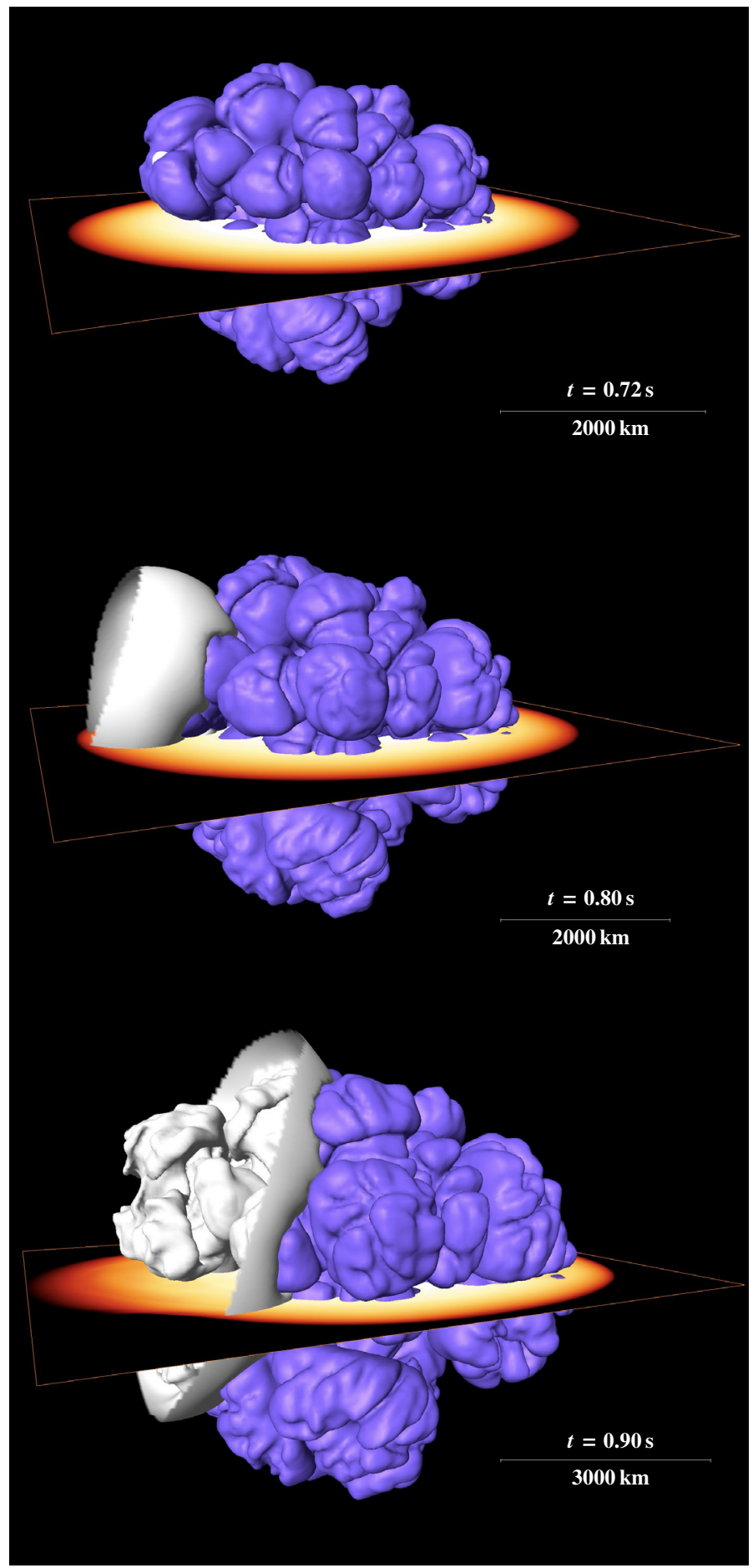

Fig. 1. Initiation and propagation of the deflagration wave (white isosurface) in Model DD_020. The deflagration flame is shown as dark isosurface and the extend of the star is indicated by the central plane mapping the logarithm of the density.

in all simulations. This is the reason for the virtually complete C-depletion in all models. The different expansion history, however, is reflected by up to $\sim 0.2 M_{\odot}$ of $\mathrm{O}$ originating from material detonating at densities less than $10^{7} \mathrm{~g} \mathrm{~cm}^{-3}$.

Although the fuel density at the location of the DDT increases with the number of deflagration ignition spots and the DDT sets in earlier, the explosions do not become stronger. The most important parameter for the overall explosion strength is the nuclear energy release in the deflagration phase prior to the DDT, $E_{\text {nuc }}^{\mathrm{DDT}}$. It accounts for the expansion of the star and therefore determines which fraction of fuel is available for the detonation to burn to NSE or IME. Obviously, the asymptotic kinetic energy is larger in models releasing less nuclear energy prior the the DDT (cf. Table 1). Moreover, the complexity of the deflagration flame increases with more ignition spots impeding and delaying the propagation of the detonation. These effects cause a larger number of ignition spots to shift the emphasis of the explosion model from the detonation phase to the deflagration phase which is characterized by the ratio of the mass of nuclear ash produced by the deflagration to that produced by the detonation, $\mathcal{R}_{\text {det }}^{\text {def }}$ (see Table 1). Whereas in Model DD_800 the deflagration mass consumption exceeds that of the detonation by a factor of 1.59, the detonation burns about three times as much material as the deflagration in Model DD_005.

This way, we obtain three different final configurations. Assuming that a large part of the NSE material is actually ${ }^{56} \mathrm{Ni}$, which by radioactive decay powers the light curve of the event, Model DD_005 would correspond to a very bright supernova. Model DD_020 provides an intermediate case with values similar to the one-dimensional W7 model (Nomoto et al. 1984), which is considered to be prototypical for many $\mathrm{SNe}$ Ia, while Model DD_800 corresponds to a dimmer event. In the latter, the detonation does not significantly enhance the NSE production over similar pure deflagration models (Röpke et al. 2006). The major difference to pure deflagration models is a more complete burning of the outer parts of the WD leading to larger asymptotic kinetic energies of the ejecta and a different composition of these. The inner core is dominated by NSE material, partially mixed with IME originating from the deflagration phase and low-density detonation burning. Above, a layer dominated by IME is found which is completely C-depleted in agreement with a recent analysis of observed spectra (Stehle et al. 2005).

The asymptotic kinetic energies of the ejecta range around the expected value of $\sim 10^{51} \mathrm{erg}$. In all tested scenarios, the DDT occurs before the deflagration structure encloses large pockets of fuel, which would be unreachable for the detonation wave. This, however, may in part be due to the low resolution of the simulations and needs further exploration.

\section{Conclusions}

In the presented study, we imposed a hypothetical DDT criterion on SN Ia explosion simulations. Being guided by a physical scenario (the transition of the deflagration flame from the flamelet to the distributed burning regime), it cannot be used to tune the models. Thus, the success of the presented simulations to reproduce gross features of SNe Ia, although it cannot be taken as a confirmation of the hypothesis, leaves the possibility of a flamedriven DDT open to a more detailed investigation of potential physical mechanisms. This result is not obvious, since the time and location of the DDT is not fixed and it thus may occur at instances disfavoring an efficient detonation. However, the three simulations presented here trigger a successful detonation after very different deflagration phases indicating robustness of the criterion. Parameterizing the DDT according to properties of the turbulent deflagration flame is naturally very sensitive to the turbulence description and we emphasize the importance of a wellfounded subgrid-scale model.

The three-dimensional simulations presented here produce somewhat less energetic explosions than the two-dimensional setups of Golombek \& Niemeyer (2005). This is most likely due to the symmetry constraints in the two-dimensional 
Table 1. Model parameters: the DDT columns provide values for the time of the initiation of the detonation wave $\left(t_{\mathrm{DDT}}\right)$, for its distance from the center of the WD, for the density at the DDT spot $\left(\rho_{\mathrm{DDT}}\right)$, and for the nuclear energy release in the deflagration prior to the DDT $\left(E_{\mathrm{nuc}}^{\mathrm{DDT}}\right)$. The last six columns characterize the asymptotic kinetic energy of the ejecta $\left(E_{\mathrm{kin}}^{\text {asympt }}\right)$ and the composition of the final stages of the explosion models, and give the ratio $\mathcal{R}_{\mathrm{det}}^{\mathrm{def}}$ of the masses of nuclear ash produced by the deflagration to that produced by the detonation.

\begin{tabular}{|c|c|c|c|c|c|c|c|c|c|c|c|}
\hline \multirow[t]{2}{*}{ Model } & \multirow{2}{*}{$\begin{array}{l}\text { Deflagration } \\
\text { ignition spots }\end{array}$} & \multicolumn{4}{|c|}{$\overline{\overline{\text { DDT }}}$} & \multicolumn{6}{|c|}{ Final stage $(t=10 \mathrm{~s})$} \\
\hline & & $\begin{array}{l}t_{\mathrm{DDT}} \\
{[\mathrm{s}]}\end{array}$ & $\begin{array}{l}\text { distance from } \\
\text { center }\left[10^{8} \mathrm{~cm}\right]\end{array}$ & $\begin{array}{l}\rho_{\text {DDT }} \\
{\left[10^{7} \mathrm{~g} \mathrm{~cm}^{-3}\right]}\end{array}$ & $\begin{array}{l}E_{\text {nuc }}^{\text {DDT }} \\
{[\text { foe] }}\end{array}$ & $\begin{array}{l}E_{\text {kin }}^{\text {asymt }} \\
\text { [foe] }\end{array}$ & $\begin{array}{l}M(\mathrm{NSE}) \\
{\left[M_{\odot}\right]}\end{array}$ & $\begin{array}{l}M(\mathrm{IME}) \\
{\left[M_{\odot}\right]}\end{array}$ & $\begin{array}{l}M(\mathrm{C}) \\
{\left[M_{\odot}\right]}\end{array}$ & $\begin{array}{l}M(\mathrm{O}) \\
{\left[M_{\odot}\right]}\end{array}$ & $\mathcal{R}_{\mathrm{det}}^{\mathrm{def}}$ \\
\hline DD_005 & 5 & 0.731 & 1.733 & 1.33 & 0.221 & 1.524 & 1.141 & 0.220 & 0.00586 & 0.0386 & 0.37 \\
\hline DD_020 & 20 & 0.724 & 1.733 & 1.92 & 0.500 & 1.237 & 0.833 & 0.435 & 0.0190 & 0.118 & 1.48 \\
\hline DD_800 & 800 & 0.675 & 1.820 & 2.40 & 0.656 & 1.004 & 0.638 & 0.547 & 0.0297 & 0.189 & 1.59 \\
\hline
\end{tabular}

simulations where the detonation wave is initiated in two toroidal rings. Moreover, deflagrations are more energetic in three dimensions and produce a more complex structure do to the additional degree of freedom, both effects hampering the detonation wave propagation. Nonetheless, almost all WD material is subject to nuclear burning in the models studied here. Yet the detonation wave does not in all cases outrun the deflagration features on the far side of the DDT. This leads to asymmetries in the structure of the outermost ejecta which, however, contain little mass. Such asymmetry effects may be an artifact of our restriction to a single DDT. The DDT criterion can easily be modified to account for multiple DDTs at each patch of the flame where the distributed burning regime is entered. Such parameters of the DDT criterion will certainly have an impact on the explosion which will be analyzed in a forthcoming study. Although we expect that the range of variation in the results will be similar to that found with a single DDT, the correlation between the number of ignition kernels and the explosion strength will possibly shift towards stronger explosions for denser ignitions.

The results of the explosion simulations are in good agreement with current observational constraints of SNe Ia. Our set of models indicates that the presented delayed detonation scenario can cover at least large parts of the observed sample of SNe Ia with respect to explosion energies and NSE material production. However, for a thorough comparison with observational results, a detailed analysis of the ejecta composition on the basis of high-resolution models is required and will be addressed in a forthcoming study.

Acknowledgements. We thank Irina Golombek for providing her implementation of the DDT and Wolfgang Hillebrandt and Paolo Mazzali for helpful discussions. The research of J.C.N. was supported by the Alfried Krupp Prize for Young University Teachers of the Alfried Krupp von Bohlen und Halbach Foundation.

\section{References}

Arnett, W. D. 1969, Ap\&SS, 5, 180

Blinnikov, S. I., Röpke, F. K., Sorokina, E. I., et al. 2006, A\&A, 453, 229

Cabot, W. H., \& Cook, A. W. 2006, Nat. Phys., 2, 562

Gamezo, V. N., Khokhlov, A. M., \& Oran, E. S. 2005, ApJ, 623, 337

Gamezo, V. N., Khokhlov, A. M., Oran, E. S., Chtchelkanova, A. Y., \& Rosenberg, R. O. 2003, Science, 299, 77

Golombek, I., \& Niemeyer, J. C. 2005, A\&A, 438, 611

Khokhlov, A. M. 1991, A\&A, 245, 114

Kozma, C., Fransson, C., Hillebrandt, W., et al. 2005, A\&A, 437, 983

Lisewski, A. M., Hillebrandt, W., \& Woosley, S. E. 2000, ApJ, 538, 831

Maier, A., \& Niemeyer, J. C. 2006, A\&A, 451, 207

Niemeyer, J. C. 1999, ApJ, 523, L57

Niemeyer, J. C., \& Hillebrandt, W. 1995, ApJ, 452, 769

Niemeyer, J. C., \& Kerstein, A. R. 1997, New Astron., 2, 239

Niemeyer, J. C. \& Woosley, S. E. 1997, ApJ, 475, 740

Nomoto, K., Sugimoto, D., \& Neo, S. 1976, Ap\&SS, 39, L37

Nomoto, K., Thielemann, F.-K., \& Yokoi, K. 1984, ApJ, 286, 644

Reinecke, M., Hillebrandt, W., \& Niemeyer, J. C. 2002a, A\&A, 386, 936

Reinecke, M., Hillebrandt, W., \& Niemeyer, J. C. 2002b, A\&A, 391, 1167

Reinecke, M., Hillebrandt, W., Niemeyer, J. C., Klein, R., \& Gröbl, A. 1999, A\&A, 347, 724

Röpke, F. K. 2005, A\&A, 432, 969

Röpke, F. K., \& Hillebrandt, W. 2005a, A\&A, 431, 635

Röpke, F. K., \& Hillebrandt, W. 2005b, A\&A, 429, L29

Röpke, F. K., Hillebrandt, W., \& Niemeyer, J. C. 2004a, A\&A, 420, 411

Röpke, F. K., Hillebrandt, W., \& Niemeyer, J. C. 2004b, A\&A, 421, 783

Röpke, F. K., Hillebrandt, W., Niemeyer, J. C., \& Woosley, S. E. 2006, A\&A, 448,1

Schmidt, W., \& Niemeyer, J. C. 2006, A\&A, 446, 627

Schmidt, W., Niemeyer, J. C., Hillebrandt, W., \& Röpke, F. K. 2006, A\&A, 450, 283

Sharpe, G. J. 1999, MNRAS, 310, 1039

Stehle, M., Mazzali, P. A., Benetti, S., \& Hillebrandt, W. 2005, MNRAS, 360, 1231

Timmes, F. X., \& Woosley, S. E. 1992, ApJ, 396

Woosley, S. E., \& Weaver, T. A. 1994, in Les Houches Session LIV: Supernovae, ed. S. Bludman, R. Mochovitch, \& J. Zinn-Justin (Amsterdam: NorthHolland), 63

Zingale, M., Woosley, S. E., Rendleman, C. A., Day, M. S., \& Bell, J. B. 2005, ApJ, 632, 1021 